\title{
Guided wave scattering analysis around a circular delamination in a quasi-isotropic fiber-composite laminate
}

Maio, L., Hervin, F., Fromme, P.

L. Maio, F. Hervin, P. Fromme, "Guided wave scattering analysis around a circular delamination in a quasi-isotropic fiber-composite laminate," Proc. SPIE 11381, Health Monitoring of Structural and Biological Systems XIV, 113810Q (23 April 2020); doi: 10.1117/12.2559125

SPIE. Event: SPIE Smart Structures + Nondestructive Evaluation, 2020, Online Only 


\title{
Guided wave scattering analysis around a circular delamination in a quasi-isotropic fiber-composite laminate
}

\author{
L. Maio ${ }^{\mathrm{a}}$, F. Hervin ${ }^{\mathrm{b}}$ and P. Fromme ${ }^{\mathrm{b}}$ \\ ${ }^{a}$ Department of Industrial Engineering, University of Naples "Federico II", Naples, Italy \\ ${ }^{\mathrm{b}}$ Department of Mechanical Engineering, University College London, WC1E 7JE, UK
}

\begin{abstract}
Carbon fiber reinforced composites are widely used in the aerospace industry, but barely visible impact damage can lead to delamination and compromise the structural integrity. The scattering of the fundamental anti-symmetric guided wave mode ( $\mathrm{A}_{0}$ Lamb mode) at an artificial circular delamination in a quasi-isotropic laminate was investigated experimentally. A 5 cycle Hanning windowed wave pulse was used as the excitation signal for the experiments. Fast Fourier Transform was employed to identify the guided wave amplitude of the scattered field along various directions. The experimental wavefield was captured using a laser Doppler vibrometer. Experimental results are presented for the scattering pattern and scattering amplitude as a function of distance from the damage. The results of this study can help to improve delamination detection techniques using guided waves and to gain physical insights into the scattering of guided waves at a delamination.
\end{abstract}

Keywords: Composite material, Laminate, Lamb waves, Scattering, Delamination.

\section{INTRODUCTION}

Composite laminates have been extensively used over the last decades in several industries to reduce the weight of structures, while improving their mechanical performance. However, composite multi-layer structures are prone to develop damage, especially when stressed by transversely concentrated loading, such as impacts. This poses new challenges for implementing structural health monitoring (SHM) techniques. Interlaminar cracking, i.e., cracking in the interfacial plane between two adjacent layers in a composite laminate, also referred to as delamination, represents the most common and critical failure mode, causing potential separation of the composite plies (laminae). This phenomenon can occur at cut (free) edges, such as at holes, or at an exposed surface through the thickness. It can also form as the result of low-velocity impacts [1,2]. In contrast to metals, in polymer composites delamination can take place under a relatively small impact load, such as that from a dropped tool, in the absence of any visible surface damage, making it difficult to detect by visual inspection $[1,3]$. The subsequent application of external loads may determine the growth of delamination cracks until it leads to a rapid deterioration of the mechanical properties, and finally catastrophic failure of the composite structure [4]. Different non-destructive techniques to detect and characterize damage and to monitor composite components include radiographic [5, 6] and ultrasonic methods [7].

Guided ultrasonic wave techniques for the rapid screening of large areas have been of considerable interest in nondestructive testing of composite structures [8], e.g., for the monitoring of bond layer stiffness of stiffeners [9]. Scattering and mode conversion at the damage are the fundamental physical effects used for the detection and characterization of structural defects or material inhomogeneities. Numerical simulations and experimental investigations have demonstrated the capacity for the detection of delaminations $[10,11]$. The reflection of the incident symmetric mode $\left(\mathrm{S}_{0}\right)$ from a delamination is strongly dependent on the position of the disbonding through the laminate thickness, and no wave reflection is obtained when the shear stress is zero at that interface [8]. Among the fundamental guided wave modes, the antisymmetric mode $\left(\mathrm{A}_{0}\right)$ was found to be more sensitive to delaminations [12]. When the $\mathrm{A}_{0}$ mode interacts with disbonds, mode conversion to $\mathrm{S}_{0}$ occurs in the sub-laminates and in the delaminated region multiple reflections occur [13]. When the depth location of the disbond is away from the mid-plane of the laminate, high amplitude modes are confined to the thin sub-laminate.

The scattering characteristics of the fundamental anti-symmetric mode at material and geometric discontinuities have been investigated numerically and experimentally. For quasi-isotropic composite laminates, the scattering directivity patterns are dominated by the fiber orientation of the outer layers and are quite different for composite laminates with the same number of laminae but different lamination sequence. In general, the $\mathrm{A}_{0}$ scattering at a delamination in a multi-layer plate

Health Monitoring of Structural and Biological Systems XIV, edited by Paul Fromme,

Zhongqing Su, Proc. of SPIE Vol. 11381, 113810Q - (C) 2020 SPIE

CCC code: $0277-786 \mathrm{X} / 20 / \$ 21 \cdot$ doi: $10.1117 / 12.2559125$

Proc. of SPIE Vol. 11381 113810Q-1 
is more complicated than the scattering at a defect in isotropic plates [14]. Scatter amplitudes and directivity distributions depend on the delamination size to wavelength ratio and the through-thickness location of the delamination damage. For a circular delamination, the amplitudes show a large forward scattered wave relative to the reflected pulse, as highlighted by numerical simulations [15]. The disbond width has a strong influence on the scattering directivity and the angular pattern of the scattered wave field is to a large degree independent of the delamination length, while the delamination depth has a significant influence on the magnitude of the scattered waves [16]. These considerations can be employed for the characterization of delamination damage in SHM applications. For mid-plane edge delamination in a quasi-isotropic composite laminate, the fiber steering effects in the outermost plies have a significant influence on the scattering pattern [17], confirming previous results [14]. This suggests that the placement of sensors along the fiber direction is optimal to detect the scattered field. The maximum amplitude in the scattered field increases with delamination radius, as already highlighted [16]. The forward scattered field becomes dominant with increase in delamination size. For the interaction of the symmetric mode $S_{0}$ at delaminations in quasi-isotropic GFRP composite laminates, the sensitivity of the $S_{0}$ mode reflection depends on the delamination position in the thickness direction and ply layup orientation of the composite laminate [18].There are limited experimental studies focused on the scattering at a circular delamination in composite laminates. In this work, full field, noncontact laser measurements of the $\mathrm{A}_{0}$ wave mode scattering at a circular delamination in a quasi-isotropic composite laminate are presented and discussed.

\section{EXPERIMENTAL MEASUREMENTS}

Guided waves by piezoelectric transducers are widely used for damage detection in thin-walled structures, due to their ability to propagate over long distances, allowing fast and efficient detection of defects in large structures [19]. Guided waves are dispersive ultrasonic waves characterized by several propagation modes. Due to increasing wave attenuation with frequency, especially for composite structures the first antisymmetric $\left(\mathrm{A}_{0}\right)$ and first symmetric $\left(\mathrm{S}_{0}\right)$ modes at lower frequencies are often used for damage detection. The $\mathrm{A}_{0}$ mode has shorter wavelengths (at the same excitation frequency) and has been found to be more suitable for the detection of small damage in composites. Therefore, in this work only the $\mathrm{A}_{0}$ guided wave mode is considered and its interaction with a circular delamination is investigated.

For the experimental study, the specimen is a symmetric, quasi-isotropic graphite/epoxy laminate [20] with dimensions $600 \times 600 \times 1.6 \mathrm{~mm}^{3}$ and stacking sequence $[+45 /-45 / 0 / 90]_{\mathrm{s}}$. The mechanical properties of the single laminae are provided in Table 1.

\begin{tabular}{c|c|c}
$\mathrm{E}_{1}$ & 175 & $\mathrm{GPa}$ \\
$\mathrm{E}_{2}$ & 6.9 & $\mathrm{GPa}$ \\
$\mathrm{E}_{3}$ & 6.9 & $\mathrm{GPa}$ \\
$\mathrm{G}_{12}$ & 4.18 & $\mathrm{GPa}$ \\
$\mathrm{G}_{13}$ & 4.18 & $\mathrm{GPa}$ \\
$\mathrm{G}_{23}$ & 2.35 & $\mathrm{GPa}$ \\
$v_{12}$ & 0.2542 & \\
$v_{13}$ & 0.2542 & \\
$v_{23}$ & 0.4689 & \\
$\rho$ & 1520 & $\mathrm{~kg} / \mathrm{m}^{3}$
\end{tabular}

Table 1. Mechanical properties of the CFRP laminae.

A circular polytetrafluoroethylene (PTFE) film, $15 \mathrm{~mm}$ in diameter, was included during the manufacturing at the second interface (i.e. between the $2^{\text {nd }}$ and $3^{\text {rd }} \mathrm{ply}$ ) to simulate an artificial disbond. Around the artificial delamination, a circular crown was identified through an ultrasonic immersion C-Scan where the plies are detached, giving an actual flaw diameter of approximately $20 \mathrm{~mm}$ [21]. A schematic view showing the laminated panel is given in Figure 1, with the location of the defect, transducer, and measurement area shown. 


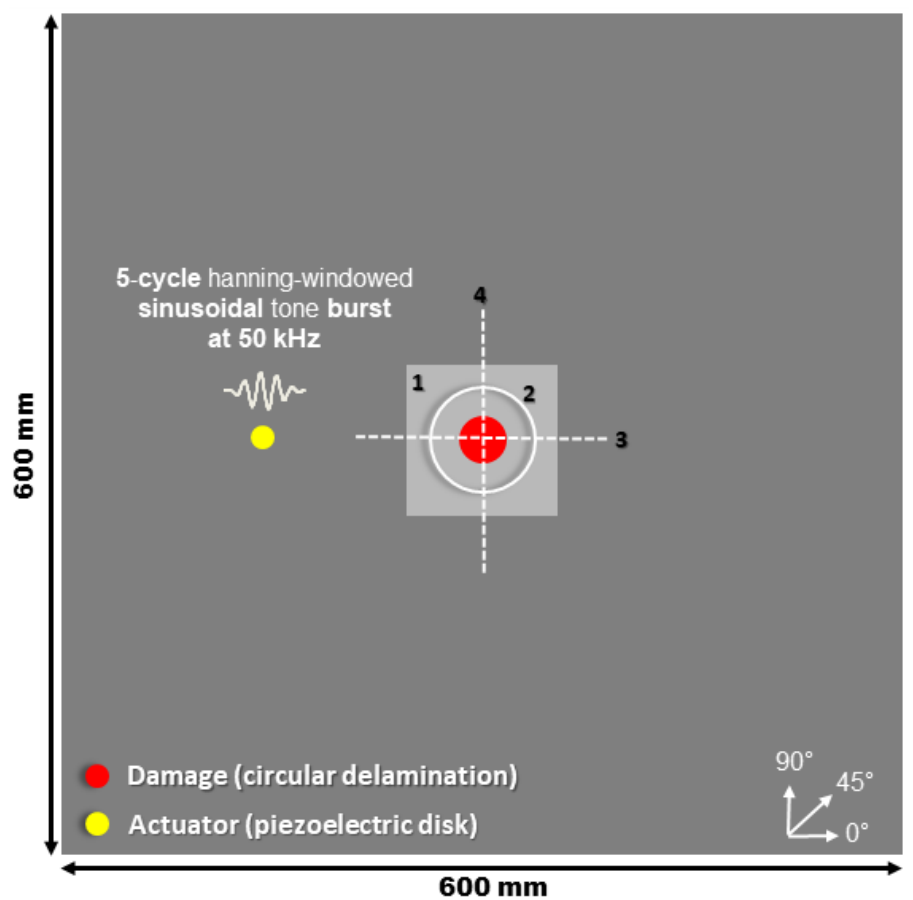

Figure 1. Schematic of quasi-isotropic composite laminate (top view) with damage and PZT actuator marked; square (1) and circle (2) show scanning area, horizontal (3) and vertical (4) dotted lines represent the scanning paths across the flaw.

The experimental setup is shown in Figure 2. A piezoelectric transducer (lead zirconate titanate (PZT) disk, PI Ceramic PIC-255, diameter $10 \mathrm{~mm}$, thickness $0.25 \mathrm{~mm}$ ) was bonded by cyanoacrylate glue to the surface of the composite plate $100 \mathrm{~mm}$ from the center of the delamination location and was used to generate the $\mathrm{A}_{0}$ guided wave mode. The excitation signal was a 5-cycle sine wave at $50 \mathrm{kHz}$ modulated by a Hanning window and was generated using a programmable function generator (Agilent 33220A).

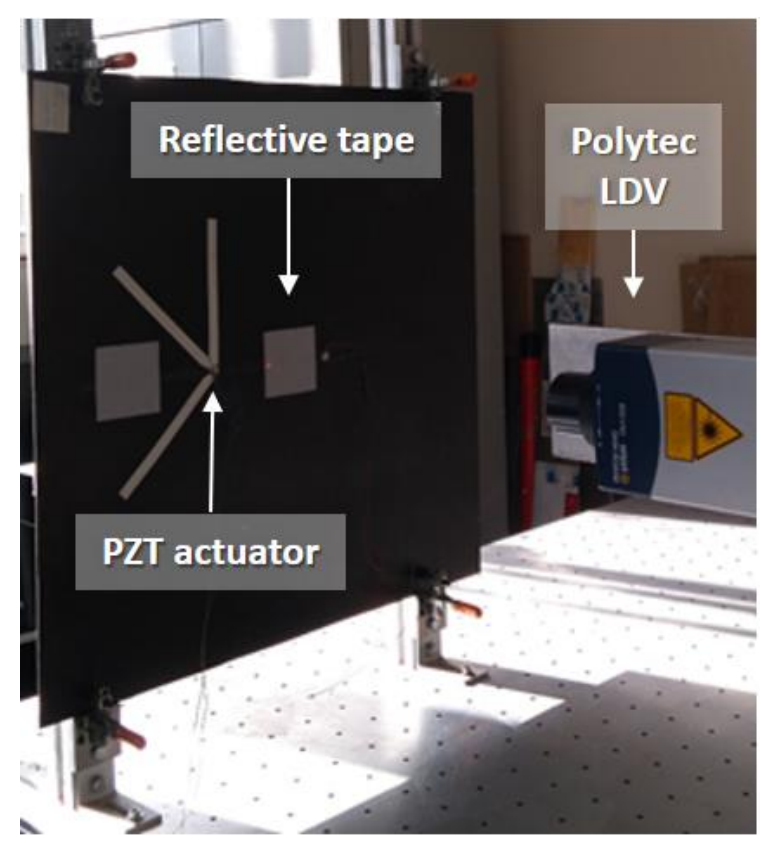

Figure 2. Experimental setup with laser head and CFRP specimen. 
The excitation signal was amplified to $50 \mathrm{Vpp}$ (Krohn-Hite 7602M wideband amplifier) and applied to the transducer. A laser vibrometer (Polytec sensor head OFV-505, OFV-5000 vibrometer controller) attached to a scanning rig was used to measure the velocity of the out-of-plane displacement of the plate surface. The laser head can move parallel to the sample both horizontally and vertically. Retroreflective tape was applied to the plate to improve the laser beam reflection and thus signal-to-noise ratio. The time signals were filtered using a $25-75 \mathrm{kHz}$ band-pass filter and recorded and averaged 20 times using a digital storage oscilloscope. The signals were then saved to a PC to be further analyzed in MATLAB.

Three different scans were performed on the sample, shown in Figure 1. A square area $40 \mathrm{~mm}$ x $40 \mathrm{~mm}$ centered on the delamination was scanned in $1 \mathrm{~mm}$ steps. A circular scan centered on the delamination with radius $30 \mathrm{~mm}$ was performed in steps of $2^{\circ}$. A linear scan $70 \mathrm{~mm}$ in length was performed horizontally, and vertically, in $1 \mathrm{~mm}$ steps with each line crossing through the central point of the delamination. Three different analyses were performed in order to obtain the maximum signal amplitude, the total pulse energy, and the magnitude of the frequency component at the center frequency of $50 \mathrm{kHz}$ using Fast Fourier transform (FFT) of the scattered wavefield. Signals were time gated to include only the reflected pulse before being analyzed. The maximum amplitude was obtained by taking the Hilbert transform to calculate the envelope of the signal and extracting the maximum value of the envelope. The total energy was obtained by summing the square of the amplitude for each point in the time trace. Amplitudes were normalized using an estimate of the incident wave amplitude as a reference value. The amplitude of the incident wave was estimated by calculating the mean of the amplitude values in front and behind the delamination from the horizontal line scan and either side of the delamination in the vertical line scan. A similar method was used to normalize the total energy of the signal.

\section{GUIDED WAVE SCATTERING AT DELAMINATIONS}

Figure 3 shows the scattered wavefield of the $40 \mathrm{~mm} \times 40 \mathrm{~mm}$ area surrounding the delamination. The incident wave propagates left to right (direction of x-axis). The maximum amplitude (Figure 3a) increases in a circular region that corresponds to approximately the size of the delamination (diameter: $20 \mathrm{~mm}$ ). The points with high amplitude suggest that energy is trapped on top of the delamination, as reported in literature. The regions of high and low amplitude within the delamination area might indicate multiple reflections of the wave modes trapped within the delamination. A forward scattered wave with higher amplitude can be observed to the right of the circular region, behind the delamination. Two regions of low amplitude either side of the forward scattered wave can be seen. This suggests that destructive interference is occurring between the incident and forward scattered wave. The scattering pattern is reasonably symmetric, which is to be expected due to the symmetric shape of the artificial insert. A similar scattering pattern can be observed in the total energy of the wavefield (Figure 3b) and the magnitude of the FFT at $50 \mathrm{kHz}$ (Figure 3c). Therefore, only the maximum amplitude evaluation will be considered for the rest of this discussion.

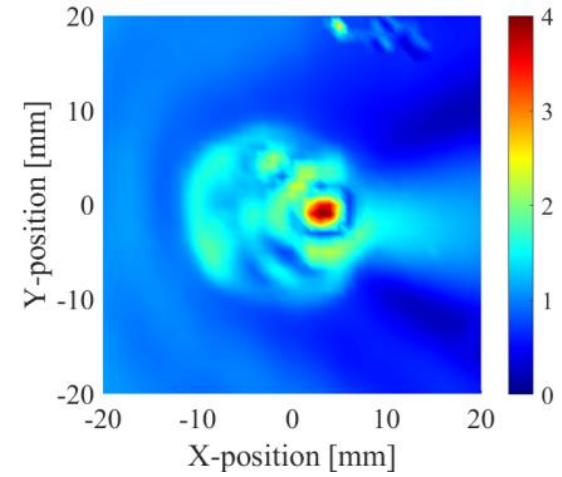

a)

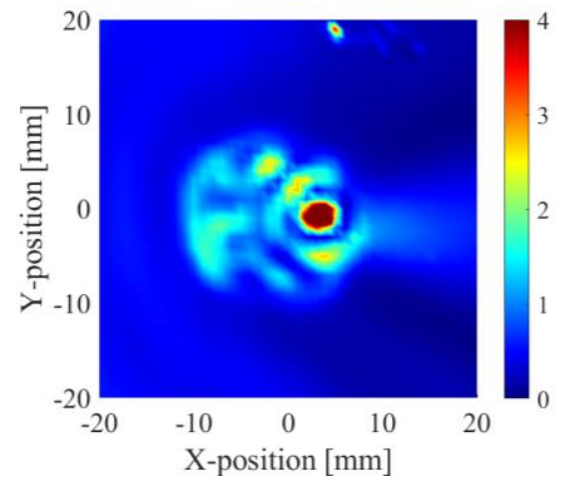

b)

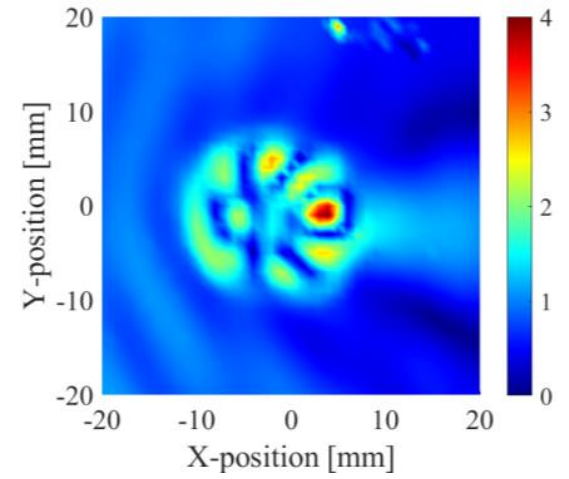

c)

Figure 3. Measured scattered wavefield of $\mathrm{A}_{0}$ mode at $50 \mathrm{kHz}$ in $40 \mathrm{~mm}$ x $40 \mathrm{~mm}$ area centered on circular delamination (diameter: $20 \mathrm{~mm}$ ); a) Normalized maximum amplitude of signal envelope; b) Normalized total energy of wave pulse. c) Normalized magnitude of FFT at $50 \mathrm{kHz}$. Position $(0,0)$ approximately corresponds to center of delamination. Incident wave propagates from left to right. 


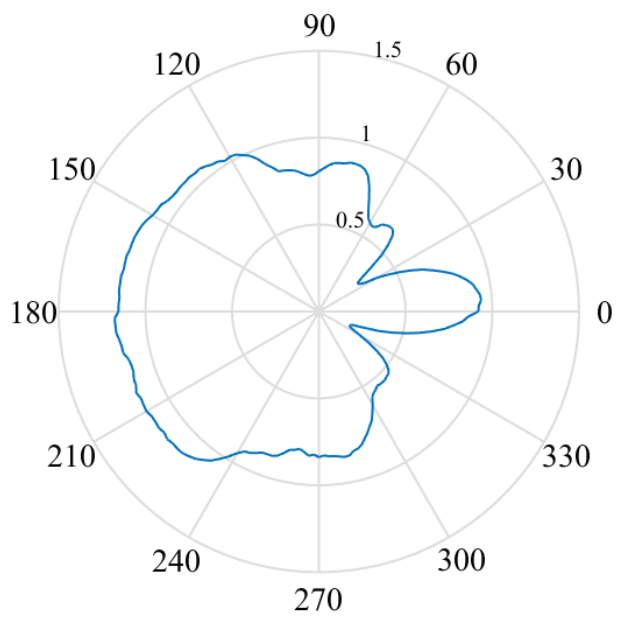

Figure 4. Measured maximum scattered amplitude (normalized) of $\mathrm{A}_{0}$ mode at $50 \mathrm{kHz}$ for circular scan (30 mm radius) around delamination. Incident wave from $180^{\circ}$ direction.

Figure 4 shows the angular maximum scattered amplitude for a circular scanning path with $30 \mathrm{~mm}$ radius centered on the delamination region. The lobe in the $0^{\circ}$ direction corresponds to the forward scattered amplitude. There is a steep drop in amplitude in the $30^{\circ}$ and $330^{\circ}$ directions, suggesting destructive interference between the incident and forward scattered waves as observed in Figure 3a. The forward scattered amplitude is generally lower than the backscattered amplitude which is highest between $120^{\circ}-240^{\circ}$. The scattering pattern is reasonably symmetric.

Figure 5 shows the maximum scattered amplitude along $70 \mathrm{~mm}$ horizontal and vertical lines passing through the center of the delamination. The amplitude decreases along the horizontal line (Figure 5a) until the delamination region is reached, where a sharp increase in amplitude is observed. This is consistent with the scanning results shown in Figure 3 . The decrease in amplitude with propagation distance outside of the delamination region is to be expected, as the distance from the transducer and thus wave attenuation is increasing. A significant decrease in amplitude can be observed at the edge of the delamination at $+7 \mathrm{~mm}$. After $+10 \mathrm{~mm}$ the forward scattered amplitude is greater than that of the incident wave, consistent with the forward scattered lobe observed above. The amplitude of the vertical line scan in Figure $5 \mathrm{~b}$ increases between $-10 \mathrm{~mm}$ to $+10 \mathrm{~mm}$ which corresponds to the delamination region. The amplitude of the scattered field is approximately symmetric on either side of the delamination region, showing some interference. The amplitude is not symmetric within the delamination for both the horizontal and vertical scans.

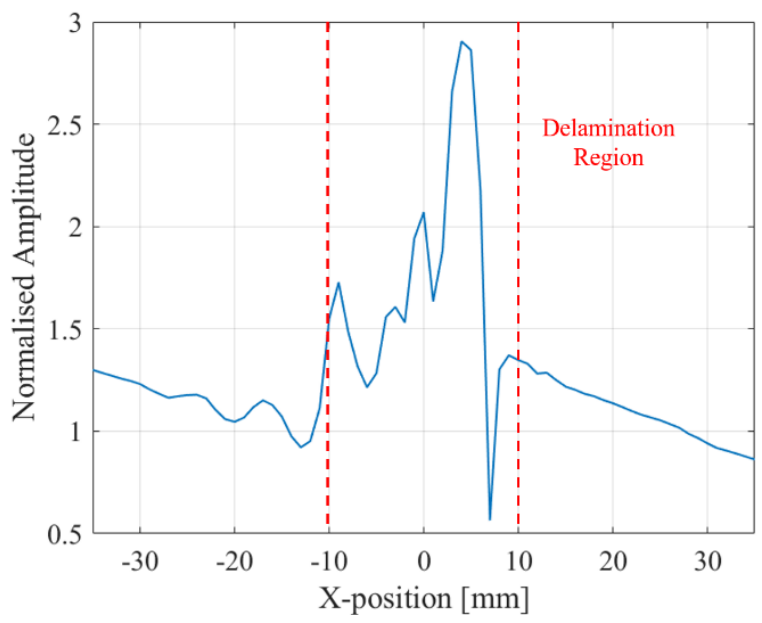

a)

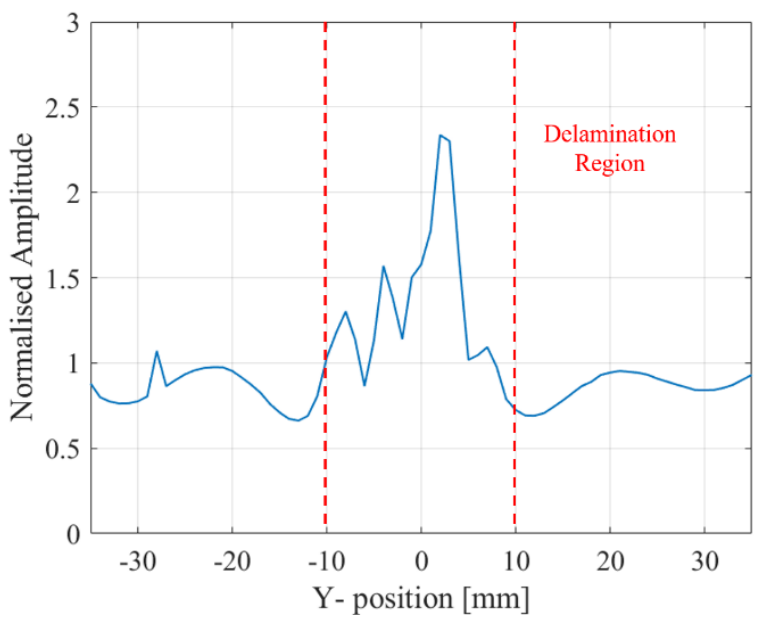

b)

Figure 5. Normalized maximum scattered amplitude for a) $70 \mathrm{~mm}$ horizontal line scan b) $70 \mathrm{~mm}$ vertical line scan. Position at $0 \mathrm{~mm}$ corresponds approximately to center of delamination; delamination region indicated by red dotted lines. 


\section{CONCLUSIONS}

The scattering of the first anti-symmetric $\mathrm{A}_{0}$ guided wave mode at an artificial circular delamination in a quasi-isotropic composite plate has been investigated systematically experimentally. A significant energy trapping within the delamination, a shadow area with low amplitude, and an energy increase in the forward scattered guided wave pulse were observed from noncontact laser measurement of the scattered wave field. The potential for impact damage detection in composites has been demonstrated and better understanding of guided wave scattering at delaminations has been obtained.

\section{ACKNOWLEDGEMENTS}

Leandro Maio wishes to express his deep and sincere gratitude to Dr Paul Fromme for the excellent opportunity to carry out research activities in his laboratory and for his valuable support throughout this work. Leandro Maio's visit to University College London was funded by the Erasmus+ Mobility Agreement for Staff Training.

\section{REFERENCES}

[1] Cantwell, W.J. and Morton, J., "Geometrical effects in the low velocity impact response of CFRP," Compos. Struct. 12, 39-59 (1989).

[2] Liu, D., "Delamination resistance in stitched and unstitched composite planes subjected to composite loading," J. Reinf. Plast. Compos. 9, 59-69 (1990).

[3] Prichard, J.C. and Hogg, P.J., "The role of impact damage in post-impacted compression testing," Compos. 21, 503$511(1990)$.

[4] Choi, N.S., Kinloch, A.J. and Williams, J.G., "Delamination fracture of multidirectional carbon-fiber/epoxy composites under mode I, mode II and mixed-mode I/II loading," J. Compos. Mater. 33, 73-100 (1999).

[5] Endrizzi, M., Murat, B.I.S., Fromme, P. and Olivo, A., "Edge-illumination X-ray dark-field imaging for visualising defects in composite structures," Compos. Struct. 134, 895-899 (2015).

[6] Shoukroun, D., Massimi, L., Iacoviello, F., Endrizzi, M., Bate, D., Olivo, A. and Fromme, P., "Enhanced composite plate impact damage detection and characterisation using X-Ray refraction and scattering contrast combined with ultrasonic imaging," Compos. B 181, 107579 (2020).

[7] Smith, R., Nelson, L., Xie, N., Fraij, C. and Hallett, S., "Progress in 3D characterisation and modelling of monolithic carbon-fibre composites," Insight 57, 131-139 (2015).

[8] Guo, N. and Cawley, P., "The interaction of Lamb waves with delaminations in composite laminates," J. Acoust. Soc. Am. 94, 2240-2246 (1993).

[9] Fan, Z., Castaings, M., Lowe, M.J.S., Biateau, C. and Fromme, P., "Feature-guided waves for monitoring adhesive shear modulus in bonded stiffeners," NDT\&E Int. 54, 96-102 (2013).

[10] Toyama, N. and Takatsubo, J., "Lamb wave method for quick inspection of impact-induced delamination in composite laminates," Compos. Sci. Technol. 64, 1293-1300 (2003).

[11] Tan, K.S., Guo, N. and Wond, B.S., "Experimental evaluation of delaminations in composite plates by the use of Lamb waves," Compos. Sci. Technol. 53, 77-84 (1995).

[12] Guy, P., Jayet, Y. and Goujon, L., "Guided wave interaction with complex delaminations. Application to damage detection in composite structures," Proc. SPIE 5047, 25-33 (2003).

[13] Ramadas, C., Balasubramaniam, K., Joshi, M. and Krishnamurthy, C.V., "Interaction of guided Lamb waves with an asymmetrically located delamination in a laminated composite plate," Smart Mater. Struct. 19, 065009 (2010).

[14] Ng, C.T. and Veidt, M., "Scattering of the fundamental anti-symmetric Lamb wave at delaminations in composite laminates," J. Acoust. Soc. Am. 129, 1288-1296 (2011).

[15] Hayat, K. and Ha, S.K., "Low-velocity impact-induced delamination detection by use of the S0 guided wave mode in cross-ply composite plates: A numerical study,” J. Mech. Sci. Technol. 28, 445-455 (2014).

[16] Murat, B.I.S., Khalili, P. and Fromme, P., "Scattering of guided waves at delaminations in composite plates," J. Acoust. Soc. Am. 139, 3044-3052 (2016).

[17] Chiu, W.K., Rose, L.R.F., Nadarajah, N., "Scattering of the fundamental anti-symmetric Lamb wave by a midplane edge delamination in a fiber-composite laminate," Proc. Eng. 188, 317-324 (2017). 
[18] Gupta, S. and Rajagopal, P., "Effect of ply orientation and through thickness position of delamination on the reflection of fundamental symmetric S0 Lamb mode in GFRP composite plate structures," Ultrasonics 90, 109-119 (2018).

[19] Hall, J.S., Fromme, P. and Michaels, J.E., "Guided Wave Damage Characterization via Minimum Variance Imaging with a Distributed Array of Ultrasonic Sensors," J. Nondestruct. Eval. 33, 299-308 (2014).

[20] Maio, L., Ricci, F., Memmolo, V., Monaco, E. and Boffa, N.D., "Application of laser Doppler vibrometry for ultrasonic velocity assessment in a composite panel with defect," Comp. Struct. 184, 1030-1039 (2018).

[21] Maio, L., Memmolo, V., Boccardi, S., Meola, C., Ricci, F., Boffa, N.D. and Monaco, E., "Ultrasonic and IR Thermographic Detection of a Defect in a Multilayered Composite Plate," Proc. Eng. 167, 71-79 (2016). 